\title{
AVALIAÇÃO DA PRODUÇÃO DE ETANOL POR DIFERENTES LEVEDURAS UTILIZANDO O HIDROLISADO ENZIMÁTICO DO BAGAÇO DE CAJU PRÉ-TRATADO COM PERÓXIDO DE HIDROGÊNIO ALCALINO
}

\author{
J.A.C. CORREIA ${ }^{1}$, A.P. BANDEIRA ${ }^{1}$, S.P. CABRAL ${ }^{1}$, L.R.B.GONÇALVES ${ }^{1}$ e M.V.P. ROCHA ${ }^{1}$. \\ ${ }^{1}$ Universidade Federal do Ceará, Departamento de Engenharia Química \\ E-mail para contato: jessyca_1905@hotmail.com; valderez.rocha@ufc.br
}

\begin{abstract}
RESUMO - Este trabalho visa estudar a produção de bioetanol a partir do bagaço de caju, matéria prima abundante no Nordeste. O pré-tratamento do bagaço de caju (BC) com peróxido de hidrogênio alcalino (PHA) e a hidrólise enzimática do Bagaço de caju prétratado (BC-PHA) foram avaliados visando a conversão de celulose e hemicelulose em açucares fermentescíveis. O BC utilizado neste estudo continha 20,56 $\pm 20,19 \%$ de celulose, $10,17 \pm 0,89 \%$ de hemicelulose e lignina 35,26 $\pm 0,90 \%$. O pré-tratamento resultou numa redução no teor de lignina dos sólidos residuais. A combinação das enzimas complexo celulase e $\beta$-glucosidase, na proporção de $0,61: 0,39$, com carga de 30 $\mathrm{FPU} / \mathrm{g}_{\mathrm{BC}-\mathrm{PHA}}$ e $66 \mathrm{CBU} / \mathrm{gBC}-\mathrm{PHA}$, respectivamente, proporcionou a maior concentração de açúcares, com rendimento de glicose $(511,68 \mathrm{mg} / \mathrm{gBC}-\mathrm{PHA}-36 \mathrm{~g}$ de glicose / L) e rendimento de xilose $(237,8 \mathrm{mg} / \mathrm{gBC}-\mathrm{PHA}$ - $13 \mathrm{~g} / \mathrm{L}$ de xilose $)$. O líquido obtido após hidrólise enzimática foi utilizado para avaliar a produção de etanol utilizando os microorganismos Saccharomyces cerevisiae, Kluyveromyces marxianus ATCC 36907 e K. marxianus CCA510 a $30{ }^{\circ} \mathrm{C}$ e $150 \mathrm{rpm}$. A concentração de etanol foi semelhante para todos os micro-organismos (aproximadamente $15 \mathrm{~g} / \mathrm{L}$ ) com coeficiente de rendimento de produto com base no consumo de substrato $\left(\mathrm{Y}_{\mathrm{P} / \mathrm{S}}\right)$ de $0,50 \mathrm{~g}_{\text {Etanol }} / \mathrm{g}_{\text {glicose }}$ para todas as cepas. No entanto, a levedura $S$. cerevisiae apresentou maior produtividade $(7,5 \pm 0,37$ $\mathrm{g} /($ L.h $))$.
\end{abstract}

\section{INTRODUÇÃO}

A tecnologia de conversão de biomassa lignocelulósica em carboidratos para posterior produção de etanol vem sendo considerada como uma das alternativas mais promissoras em substituição aos combustíveis derivados do petróleo (Hasunuma e Kondo (2012)).O seguinte trabalho tem por objetivo o aproveitamento da biomassa lignocelulósica oriunda da agroindústria do caju. Segundo dados da FAOSTAT, Organização das Nações Unidas para Alimentação e Agricultura, o Brasil é o quinto maior produtor mundial de castanha de caju com a produção de mais de 230 mil toneladas em 2011. Para a produção de bebidas, o pedúnculo é processado e resultando em $15 \%(\mathrm{~m} / \mathrm{m})$ de bagaço, o que essencialmente não tem valor comercial e é geralmente descartado pela indústria local (Rodrigues et al., 2011). Foi realizado, no presente trabalho, o pré-tratamento do bagaço de caju utilizando peróxido de hidrogênio alcalino. Segundo 
Karagöz et al. (2012) e Selig et al. (2009), o peróxido de hidrogênio alcalino atua na redução da cristalização da celulose e com a ação oxidativa dos radicais derivados do peróxido, ocorre elevada despolimerização e solubilização da lignina. A hidrólise enzimática trata-se do processo de conversão do material lignocelulósico em açúcares para uma posterior fermentação (Balat, 2011). Diversas enzimas e parâmetros são avaliadas quanto a capacidade de conversão em açúcares fermentescíveis (McIntosh e Vancov, 2011)

Nesse contexto, este trabalho objetiva o estudo do pré-tratamento do bagaço de caju com peróxido de hidrogênio alcalino para expor a estrutura recalcitrante do material lignocelulósico ao ataque enzimático. Posteriormente, foram avaliados alguns parâmetros na hidrólise enzimática, tais como a carga de enzimas e a carga de celulose, visando um maior rendimento de glicose e xilose para serem utilizados na produção de etanol pelas leveduras Saccharomyces cerevisiae, Kluyveromyces marxianus ATCC 36907 e Kluyveromyces marxianus CCA 510.

\section{MATERIAL E MÉTODOS}

\subsection{Material}

Material lignocelulósico: O Bagaço de Caju (BC) utilizado neste estudo foi gentilmente cedido pela Indústria de Processamento de Sucos Jandaia no Ceará, Brasil. O BC foi lavado três vezes com água e seco a $60{ }^{\circ} \mathrm{C}$ por $24 \mathrm{~h}$, triturado, peneirado e estocado até seu uso a temperatura ambiente. Após peneiramento do material, as partículas que ficaram retidas entre as peneiras de Mesh 20-80 (0,25-0,84 $\mathrm{mm})$ foram utilizadas como matéria-prima para a realização dos experimentos.

Enzimas: As enzimas, complexo celulase (NS22074) e $\beta$-glucosidase (NS50010), foram gentilmente fornecidas pela Novozymes (Novozyme, Bagsvaerd, Denmark). A atividade enzimática do complexo celulase e $\beta$-glucosidase foram determinadas de acordo com Ghose (1987). O teor de proteínas dos coquetéis enzimáticos foi determinado utilizando o método de Bradford (Bradford, 1976). A atividade enzimática e a concentração de proteína dos coquetéis utilizados estão apresentadas na Tabela 1.

Tabela 1 - Atividade enzimática e a concentração de proteínas das enzimas comerciais utilizadas no estudo de hidrólise do caju do bagaço pré-tratado com peróxido de hidrogênio

\begin{tabular}{|c|c|c|}
\hline Enzima & Atividade Enzimática & $\begin{array}{c}\text { Proteína } \\
\left(\mathrm{mg} / \mathrm{ml}_{\text {Extrato }}\right)\end{array}$ \\
\hline Complexo celulase & $108,12 \mathrm{FPU} / \mathrm{ml}^{\mathrm{i}}$ & 28,47 \\
\hline$\beta$-glucosidase & $384,28 \mathrm{CBU} / \mathrm{ml}^{\mathrm{ii}}$ & 28,29 \\
\hline
\end{tabular}

\subsection{Métodos}

Pré-tratamento do bagaço de caju com peróxido de hidrogênio alcalino: O bagaço de caju foi pré-tratado com peróxido de hidrogênio alcalino (PHA) utilizando uma concentração de 4,3\% v/v, em 


\section{9 a 22 de outubro de 2014 \\ Florianópolis/SC}

agitador orbital a $250 \mathrm{rpm}, 35^{\circ} \mathrm{C}$ por 6 horas. A metodologia utilizada segue o melhor resultado obtido por Correia et al.(2013).

Caracterização da matéria-prima: O bagaço de caju (BC) antes e após o pré-tratamento (BC-PHA) com peróxido de hidrogênio alcalino, foi caracterizado quanto à sua composição de celulose, hemicelulose e lignina segundo a metodologia de Gouveia (2009). Também, realizou-se análise de extraíveis conforme descritos no protocolo NREL/TP-510-42619(Sluiter et al., 2008a) e a determinação de sólidos totais e cinza segundo NREL/TP-510-42621 (Sluiter et al., 2008b).

Hidrólise Enzimática: A hidrólise enzimática do bagaço de caju pré-tratado foi realizada segundo a metodologia escrita no protocolo da NREL/TP-510-42629 (Selig et al., 2008) com algumas modificações. Uma quantidade de sólidos lavados referente a $0,3 \mathrm{~g}$ de celulose em 30 $\mathrm{mL}$ foi adicionada em frascos Erlemneyers de $150 \mathrm{~mL}$. Em cada frasco, adicionou-se 15,0 mL de tampão citrato de sódio $0,1 \mathrm{M}$, pH 4.8 e $120 \mu \mathrm{L}$ de tetraciclina $(10 \mathrm{mg} / \mathrm{mL}$ em $70 \% \mathrm{v} / \mathrm{v}$ em etanol) para prevenir o crescimento microbiano durante a hidrólise. Após, adicionou-se a quantidade de enzima suficiente para se obter a atividade desejada e calculou-se a quantidade de água necessária para completar o volume reacional de $30 \mathrm{~mL}$. Todas as soluções e material sólido foram assumidos apresentar uma densidade específica de 1,00 g/mL. Posteriormente, os frascos foram postos em agitador orbital (Tecnal - TE 422) sob agitação de $150 \mathrm{rpm}$, a $45{ }^{\circ} \mathrm{C}$ por 72 horas. A cada 24 horas, retirou-se $1,0 \mathrm{~mL}$ da mistura reacional e centrifugou-se a $10.000 \mathrm{~g}$ durante 15 minutos e os sobrenadantes foram usados para a análise de açúcares.Após a quantificação dos açúcares na amostra, determinou-se os rendimentos de glicose e xilose segundo Correia et al.(2013).

Efeito da carga enzimática na hidrólise enzimática do BC-AHP: O efeito da carga de enzima na hidrólise enzimática foi estudada com variação das cargas enzimáticas do complexo celulase e $\beta$-glucosidase de 5,0 FPU/g $\mathrm{g}_{\text {BC-PHA }}$ e 11,0 CBU/g $\mathrm{g}_{\mathrm{BC}-\mathrm{PH}}$ para $35 \mathrm{FPU} / \mathrm{g}_{\mathrm{BC}-\mathrm{PHA}}$ e $77 \mathrm{CBU} /$ $\mathrm{g}_{\mathrm{BC}-\mathrm{PHA}}$, respectivamente.

Efeito da carga de celulose na hidrólise enzimática do BC-AHP: Com o intuito de obter uma maior concentração de açúcares para posterior conversão em etanol, avaliou-se o efeito da carga de celulose $(1,0 ; 2,0 ; 3,0$ e 4,0 g de celulose/100 mL) na sacarificação do BC-PHA, fixando a melhor carga enzimática obtida nos estudos anteriores.

Fermentação do hidrolisado de BC-PHA para a produção de etanol: Avaliou-se as leveduras Saccharomyces cerevisiae, Kluyveromyces marxianus ATCC 36907 e K. marxianus CC510 na produção de etanol. Para a propagação do inoculo, foi realizada a metodologia citada por Rodrigues et al. (2011). O hidrolisado obtido após a hidrólise enzimática do BC-PHA, foi utilizado como meio de cultivo, sendo suplementado com $\left(\mathrm{NH}_{4}\right)_{2} \mathrm{SO}_{4}(1,0 \mathrm{~g} / \mathrm{L})$ e extrato de levedura $(5,0 \mathrm{~g} / \mathrm{L}) . \mathrm{O} \mathrm{pH}$ deste meio foi ajustado para 4,5 e esterilizados a $110^{\circ} \mathrm{C}$ por $10 \mathrm{~min}$. As fermentações foram conduzida em Erlenmeyer de $250 \mathrm{~mL}$ com $50 \mathrm{~mL}$ de meio sob agitação a 30 ${ }^{\circ} \mathrm{C}$ e $150 \mathrm{rpm}$ em agitador orbital (TECNAL, TE-420) com a concentração inicial de microorganismo de 1,0 $\pm 0,1 \mathrm{~g} / \mathrm{L}$. Os ensaios foram feitos em triplicata e amostras dos meios de cultivo 


\section{9 a 22 de outubro de 2014 \\ Florianópolis/SC}

$(1,0 \mathrm{~mL})$ foram coletadas em intervalos de tempo pré-definidos $(0$ a $10 \mathrm{~h})$ e submetidas à análise de biomassa, glicose, xilose e etanol.

Concentração de biomassa, glicose, xilose e etanol: A concentração celular foi determinada através da análise da densidade ótica (DO) e a concentração de biomassa, expressa em $\mathrm{g} / \mathrm{L}$, foi obtida por curva de calibração para cada micro-organismo. A densidade ótica foi acompanhada por espectrofotometria a $600 \mathrm{~nm}$ para a levedura do gênero Saccharomyces e a $660 \mathrm{~nm}$ para as leveduras do gênero Kluyveromyces. A concentração dos carboidratos (glicose e xilose) e etanol foram medidas através de Cromatografia Líquida de Alta Eficiência, em um sistema de CLAE (Waters, Milford, MA, EUA) equipado com um detector de índice de refração Waters 2414 e coluna Aminex HPX-87H a $65^{\circ} \mathrm{C}$, sendo o eluente $\mathrm{H}_{2} \mathrm{SO}_{4}$ a $5 \mathrm{mmoL} / \mathrm{L}$ e vazão de $0,5 \mathrm{~mL} / \mathrm{min}$.

\section{RESULTADOS E DISCUSSÃO}

\subsection{Caracterização do bagaço de caju in natura e após pré-tratamento com PHA}

A composição do caju bagaço in natura utilizado no presente estudo foi de $20,6 \pm 2,2 \%$ $\mathrm{m} / \mathrm{m}$ de celulose, $10,2 \pm 0,9 \% \mathrm{~m} / \mathrm{m}$ de hemicelulose, $35,3 \pm 0,9 \% \mathrm{~m} / \mathrm{m}$ de lignina, $7,8 \pm 0,6 \%$ $\mathrm{m} / \mathrm{m}$ de extraíveis e $1,6 \pm 0,1 \% \mathrm{~m} / \mathrm{m}$ de cinzas. Após o pré-tratamento usando PHA 4,3\% v/v $(\mathrm{pH}$ $11,5)$ a $35^{\circ} \mathrm{C}$ e $250 \mathrm{rpm}$ por 6 horas e uma carga de sólidos de $5 \% \mathrm{~m} / \mathrm{v}$, a composição do BC prétratado foi $44,2 \pm 0,3 \% \mathrm{~m} / \mathrm{m}$ de celulose, $18,3 \pm 0,9 \% \mathrm{~m} / \mathrm{m}$ de hemicelulose, $2,9 \pm 0,1 \% \mathrm{~m} / \mathrm{m}$ de lignina, $4,9 \pm 0,3 \% \mathrm{~m} / \mathrm{m}$ de extraíveis e $5,3 \% \pm 0,9 \% \mathrm{~m} / \mathrm{m}$ de cinzas. O pré-tratamento com PHA resultou numa diminuição do teor de lignina presente na fibra, indicando que uma fração significativa da lignina foi removida ( $91,7 \%$ de remoção). Com a diminuição do teor de lignina, a porcentagem de celulose e hemicelulose aumentou de 20,56 e $10,17 \% \mathrm{~m} / \mathrm{m}$ para 44,16 e $18,27 \%$ $\mathrm{m} / \mathrm{m}$, respectivamente. Segundo Cao et al. (2012) e Karagöz et al. (2012), o pré-tratamento com peróxido de hidrogênio alcalino aumenta a eficiência na hidrólise enzimática por deslignificação e pré-tratamento com PHA é mais eficaz para a solubilização da lignina do que o pré-tratamento alcalino (Chen et al., 2008).

\subsection{Estudo da carga enzimática}

As hidrólises e seus parâmetros foram estudados com a enzima celulase complex e a $\beta$ glucosidase, visto que a enzima $\beta$-glucosidase atua de forma conjunta com a celulase, convertendo celobiose em glicose e evitando assim a inibição da celulase (Keshwani, 2009).

Com a proporção 0,61:0,39 (complexo celulase: $\beta$-glucosidase), estudou-se o efeito das cargas dessas enzimas sobre a produção de glicose e xilose utilizando o BC-PHA e os resultados estão apresentados na Tabela 2. O estudo da carga enzimática é considerada como um dos fatores mais importantes na produção de etanol de materiais lignocelulósicos. A proporção foi calculada com base na quantidade de proteína presente no ensaio em (mg proteína/g celulose), levando em consideração a quantidade de proteína presente em $1 \mathrm{ml}$ de extrato enzimático, atividade por $\mathrm{ml}$ de extrato para cada enzima e a quantidade de bagaço para se obter $1 \mathrm{~g}$ celulose. 
Observa-se na Tabela-2 que com o aumento da carga enzimática, ocorreu uma aumento no rendimento e concentração dos açúcares. As cargas enzimáticas utilizadas nos ensaios 03, 04 e 05, possibilitam maiores rendimentos, logo maiores concentrações de glicose e xilose. Visando a produção de etanol, maiores concentrações desses açúcares no meio que será utilizado como meio fermentativo, maior será a concentração de etanol. Porém, os rendimentos de glicose obtidos nos ensaios 03, 04 e 05, não são significativamente diferentes, de acordo com análise de variância, com $p<0,05$. Aumentando a carga de enzima de 0,2 vezes (de 30 FPU/66 CBU a 35 FPU/77 CBU), não conseguiu aumentar o rendimento de açúcar final, o que sugere que a carga de celulase e $\beta$-glicosidase pode ter alcançado o ponto de saturação. Portanto, as cargas enzimática utilizadas no ensaio 04 (30 $\mathrm{FPU} / \mathrm{gBC}$-PHA complexo celulase e $66 \mathrm{CBU} / \mathrm{g}_{\mathrm{BC}-\mathrm{PHA}} \beta$-glucosidase) foram escolhidas para o processo de hidrólise do BC-PHA. Com o aumento da carga enzimática para as condições do ensaio 05, por exemplo, aumentaria mais o custo com enzimas e influenciaria no custo com a produção de etanol.

Então, para o próximo estudo, utilizou-se a proporção de 0,61:0,39, com carga enzimática de 30 FPU/g $g_{\text {BC-PHA }}$ e 66 CBU/g $g_{\text {BC-PHA }}$ para as enzimas celulase e $\beta$-glucosidase, respectivamente.

Tabela 2 - Efeito das cargas enzimática da combinação das enzimas celulase e $\beta$-glucosidade, na proporção de $0,61: 0,39$, no rendimento e concentração de glicose, xilose obtidos da hidrólise enzimática de BC-PHA a $45^{\circ} \mathrm{C}, 150 \mathrm{rpm}$ durante 72 horas.

\begin{tabular}{|c|c|c|c|c|c|c|}
\hline \multirow[b]{2}{*}{ Ensaio } & \multicolumn{2}{|c|}{ Carga de Enzimas } & \multicolumn{2}{|c|}{ Glicose } & \multicolumn{2}{|c|}{ Xilose } \\
\hline & $\begin{array}{c}\text { Celulase } \\
\left(\text { FPU/g } \text { BC-PHA }_{\text {BC }}\right)\end{array}$ & $\underset{\left(\mathrm{CBU} / \mathrm{g}_{\mathrm{BC}-\mathrm{PHA}}\right)}{\beta \text {-glucosidase }}$ & $\begin{array}{c}\text { Rendimento } \\
(\%)\end{array}$ & $\begin{array}{c}\text { Concentração } \\
(\mathrm{g} / \mathrm{L})\end{array}$ & $\begin{array}{c}\text { Rendimento } \\
(\%)\end{array}$ & $\begin{array}{c}\text { Concentração } \\
(\mathrm{g} / \mathrm{L})\end{array}$ \\
\hline 01 & 5,0 & 11,0 & $76,2 \pm 0,9$ & $6,9 \pm 0,1$ & $33,1 \pm 0,1$ & $2,9 \pm 0,0$ \\
\hline 02 & 15,0 & 33,0 & $85,8 \pm 0,7$ & $7,7 \pm 0,3$ & $64,8 \pm 0,3$ & $5,7 \pm 0,2$ \\
\hline 03 & 20,0 & 44,0 & $98,15 \pm 0,6$ & $8,8 \pm 0,2$ & $90,7 \pm 0,1$ & $7,9 \pm 0,1$ \\
\hline 04 & 30,0 & 66,0 & $103,0 \pm 1,7$ & $9,3 \pm 0,0$ & $95,3 \pm 2,1$ & $8,3 \pm 0,3$ \\
\hline 05 & 35,0 & 77,0 & $103,8 \pm 1,1$ & $10,0 \pm 0,1$ & $100,0 \pm 1,6$ & $9,0 \pm 0,2$ \\
\hline
\end{tabular}

\subsection{Estudo da carga de celulose}

Um dos principais fatores que afetam a taxa de produção de açúcares por hidrólise enzimática é a concentração inicial de substrato (de celulose e/ou hemicelulose). Altas concentrações de substrato podem causar inibições, o que reduz substancialmente a eficiência do biocatalisador. Para avaliar esse efeito, realizou-se um ensaio variando a carga inicial de celulose, ou seja, a concentração inicial de BC-PHA. A Figura 1 apresenta a concentração de glicose obtida no hidrolisado variando a carga de celulose, utilizando as condições enzimáticas do Ensaio 04 (celulase $30 \mathrm{FPU} / \mathrm{g}_{\mathrm{BC}-\mathrm{PHA}}, \beta$-glucosidase $\left.66 \mathrm{CBU} / \mathrm{g}_{\mathrm{BC}-\mathrm{PHA}}\right)$.

Analisando a Figura 1, observa-se um aumento na concentração de açúcares com o aumento da

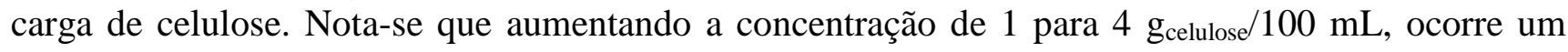


aumento de $260 \%$ na concentração de glicose, correspondendo a uma concentração de $36 \mathrm{~g} / \mathrm{L}$ com a maior carga de celulose avaliada. Aumento semelhante foi obtido para a concentração de xilose (225\%), corresponde a maior concentração de $13 \mathrm{~g} / \mathrm{L}$. Para o material lignocelulósico avaliado, observou-se um inchamento com concentrações maiores que $10 \% \mathrm{~m} / \mathrm{v}$, correspondendo a 4,2 $\mathrm{g}_{\text {celulose }} / 100 \mathrm{~mL}$ de meio reacional, por isso não se realizou ensaios com cargas maiores.

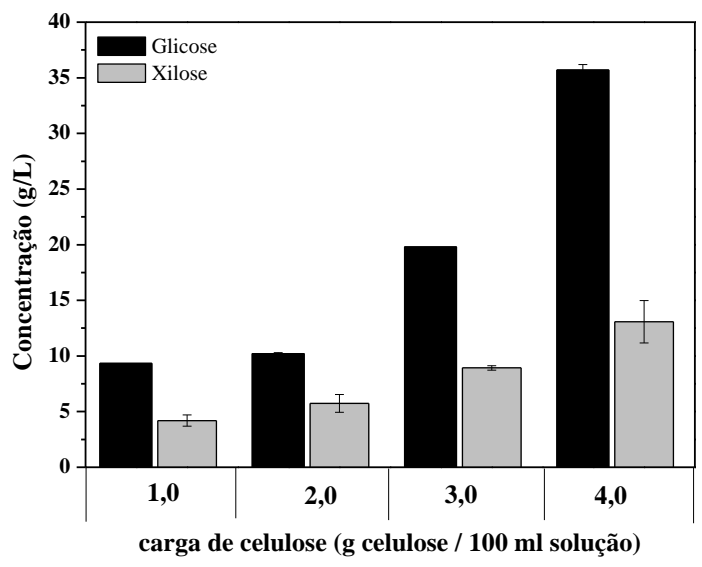

Figura 1- Efeito da carga de celulose na concentração de açúcares obtidos por hidrólise enzimática $\left(45^{\circ} \mathrm{C}, 150 \mathrm{rpm}\right.$ por $\left.72 \mathrm{~h}\right)$ do BC-PHA, usando $30 \mathrm{FPU} / \mathrm{g}_{\mathrm{BC}-\mathrm{PHA}}$ de celulase e $66 \mathrm{CBU} / \mathrm{g}_{\mathrm{BC} \text { - }}$ PHA de $\beta$-glucosidase.

Com base neste resultados, a carga de celulose de $4 \mathrm{~g} / 100 \mathrm{ml}$, foi selecionada para a hidrólise enzimática do BC-PHA e o hidrolisado obtido utilizado no estudo de produção de etanol. Outros autores (Vasquez et al., 2007) também observaram que o aumento da percentagem de sólidos teve um efeito positivo sobre a produção de glucose quando se utiliza o bagaço de cana.

\subsection{Fermentação do hidrolisado de BC-PHA para a produção de etanol (HFS)}

Os perfis de glicose, xilose e etanol são apresentados nas Figuras 2A, 2B e 2C, para os microorganismos $S$. cerevisiae, K. marxianus ATCC36907 e $K$.marxianus CCA510, respectivamente. O substrato utilizado nesse processo foi o hidrolisado enzimático obtido com as melhores condições obtidas no estudo de hidrólise do BC-PHA. Observa-se por análise das Figura 2A, 2B e 2C que os perfis de glicose, xilose e etanol para os três micro-organismos avaliados, atingem patamares semelhantes, porém a levedura $S$. cerevisiae metabolisa mais rápido a glicose, comparado com a $K$. marxianus ATCC36907 e CCA510. No estudo realizado com S. cerevisiae, a xilose não foi metabolisada e com $2 \mathrm{~h}$ a concentração de glicose no meio atingiu $0 \mathrm{~g} / \mathrm{L}$. A maior concentração de etanol (15 \pm 0,74 g.L-1) foi obtida nesse período de cultivo com uma produtividade de 7,5 $\pm 0,37$ g/(L.h) para $S$. cerevisiae. As duas leveduras $K$. marxianus consumiram a xilose, no entanto a concentração de etanol obtida foi a mesma no ensaio fermentativo conduzido com S. cerevisiae, aproximadamente $15 \mathrm{~g} / \mathrm{L}$. Porém, o metabolismo dessas cepas é mais lento e esse patamar na concentração de etanol foi obtido com 6 h de cultivo, com produtividade de 3,75 g/L.h. Então, a 
fermentação com S. cerevisiae apresenta maior produtividade em comparação com outros microorganismos estudados.O coeficiente de rendimento de produto com base no consumo de substrato ( $\mathrm{Y}_{\mathrm{P} / \mathrm{S}}$ ) foi $0,50 \mathrm{~g}_{\text {etanol }} / \mathrm{g}_{\mathrm{glicose}}$ para todas as cepas, valor próximo ao rendimento teórico 0,51. Esses resultados indicam que o hidrolisado obtido pela hidrólise enzimática do BC-PHA pode ser utilizado com substrato para a produção de etanol utilizando os três micro-organismos avaliados.
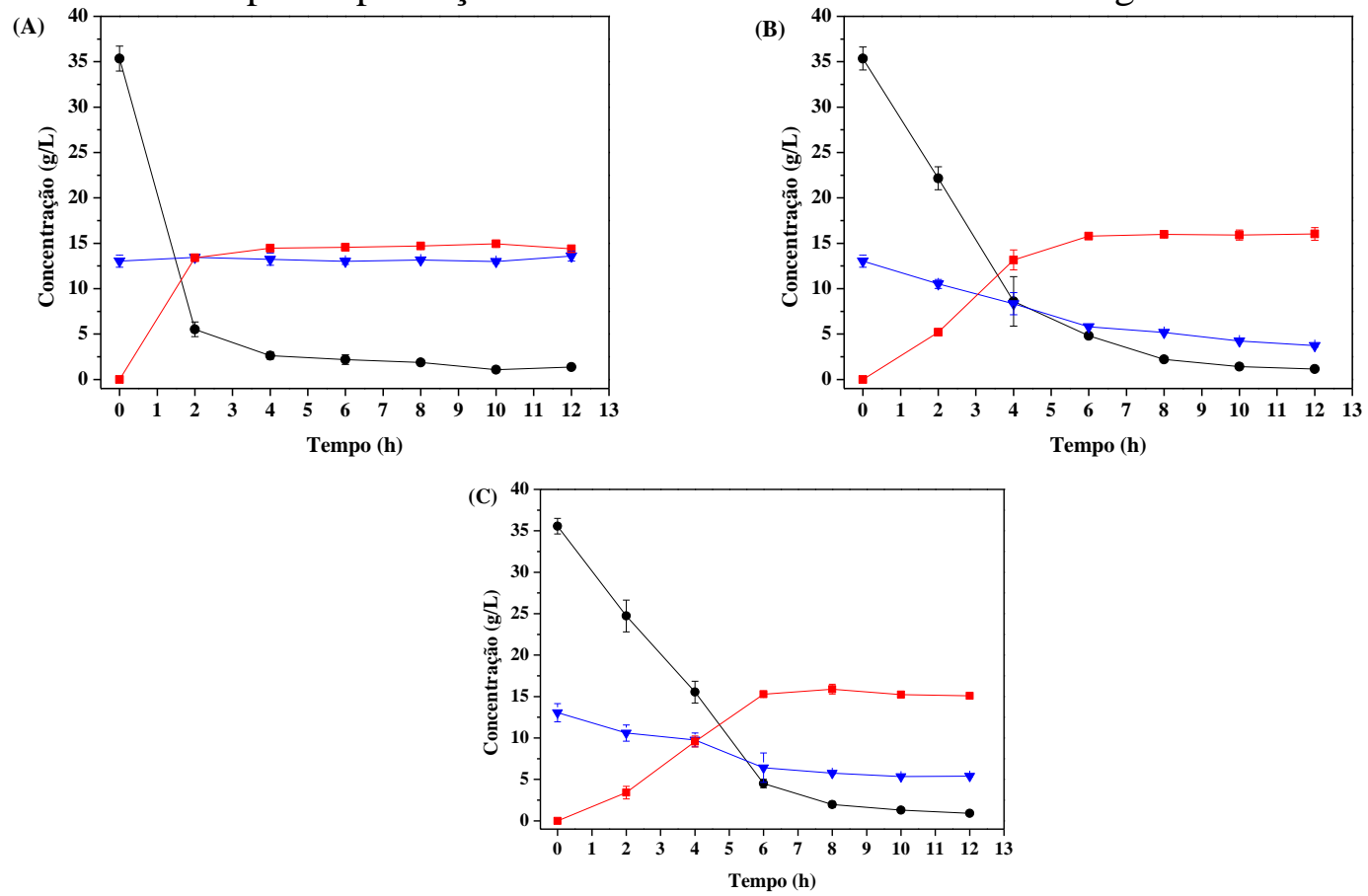

Figura 2-Produção de etanol conduzida a $30^{\circ} \mathrm{C}, 150 \mathrm{rpm}$, com o hidrolisado obtido da melhor condição de hidrólise enzimática (30 FPU/g $\mathrm{g}_{\mathrm{BC}-\mathrm{PHA}}$ complexo celulase, $66 \mathrm{CBU} / \mathrm{g}_{\mathrm{BC}-\mathrm{PHA}} \beta$-glucosidase, $4 \mathrm{~g}_{\text {celulose }} / 100 \mathrm{~mL}, 150 \mathrm{rpm}, 45^{\circ} \mathrm{C}$ por 72 horas) utilizando os micro-organismos (A) S. cerevisiae, (B)

K. marxianus ATCC36907 e (C) K. marxianus CCA510. (•) Glicose; ( $\boldsymbol{\nabla}$ ) Xilose e ( $\square$ ) Etanol.

\section{Conclusões}

Resultados obtidos mostraram que a fibra do caju apresenta potencial em carboidratos para produção de etanol por fermentação. O pré-tratamento com PHA possibilitou uma uma deslignificação do BC e após estudos de parâmetros da hidrólise enzimática, a carga enzimática com 30 FPU/g BC-AHP do complexo celulase e $66 \mathrm{CBU} / \mathrm{g}$ g BC-AHP de $\beta$-glucosidase e 4,0 g celulose / 100 solução proporcionou o melhor um rendimento de açúcar. Com o hidrolisado contendo 35,7 g/L glicose e $13 \mathrm{~g} / \mathrm{L}$ xilose, foi realizada uma fermentação que resultou resultou em um coeficiente de rendimento de produto com base no consumo de substrato $\left(\mathrm{Y}_{\mathrm{P} / \mathrm{S}}\right)$ de $0,50 \mathrm{~g}_{\text {etanol }} / \mathrm{g}_{\mathrm{glicose}}$ valor próximo ao rendimento teórico 0,51

\section{Referências bibliográficas}


BRADFORD, M. M, A rapid and sensitive method for the quantitation of microgram quantities of protein utilizing the principle of protein-dye binding, Analytical Biochemistry, v. 72, n. 7, p. 248-254, 1976.

CORREIA, J. A.C.; JUNIOR, J. E. M.; GONÇALVES, L. R. B.; ROCHA, M. V. P. Alkaline hydrogen peroxide pretreatment of cashew apple bagasse for ethanol production: Study of parameters. Bioresource Technology. v.139, p.249-256, 2013.

CHEN, H.; HAN, Y.; XU, J. Simultaneous saccharification and fermentation of steam exploded wheat straw pretreated with alkaline peroxide.Process Biochemistry, v.43, p. 1462-1466, 2008.

GOUVEIA, E. R.; DO NASCIMENTO, R. T; SOUTO-MAIOR, A. M.; ROCHA, G. J. M.

Validação de metodologia para a caracterização química de bagaço de cana-de-açúcar. Química Nova, v. 32, n. 6, p. 1500-1503, 2009.

GHOSE, T. K., Measurement of cellulose activities (recommendation of commission on biotechnology IUPAC), Pure Applied Chemistry, v. 59, p.257-268, 1987.

HASUNUMA T.; KONDO A. Consolidated bioprocessing and simultaneous saccharification and fermentation of lignocellulose to ethanol with thermotolerant yeast strains, Process Biochemistry, v. 47, p. 1287-1294, 2012.

KARAGÖZ, P.; ROCHA, I. V.; ÖZKAN, M.; ANGELIDAKI, I. Alkaline peroxide pretreatment of rapeseed straw for enhancing bioethanol production by Same Vessel Saccharification and CoFermentation. Bioresource Technology., v.104, p. 348-357, 2012.

KESHWANI, D. R. Microwave pretreatment of switchgrass for bioethanol production.2009.

Tese (Doutorado). Universidade Estadual da Carolina do Norte, Carolina do Norte, Estados Unidos da América.

MCINTOSH, S.; VANCOV, T. Optimisation of dilute alkaline pretreatment for enzymatic saccharification of wheat straw.Biomass and Bioenergy, v.35, p. 3094-3103, 2011.

RODRIGUES, T. H. S.; ROCHA, M. V. P.; MACEDO, G. R.; GONÇALVES, L. R. B. Ethanol Production from Cashew Apple Bagasse: Improvement of Enzymatic Hydrolysis by MicrowaveAssisted Alkali Pretreatment. Appl Biochem Biotechnol., v. 164, p. 929- 943, 2011.

SELIG, M.; WEISS, N.; JI Y. Enzymatic Saccharification of Lignocellulosic Biomass, Laboratory Analytical Procedure (LAP) - Technical Report, NREL/TP-510-42629, 2008.

SLUITER, A.; HAMES, B.; RUIZ, R.; SCARLATA, C.; SLUITER, J.; TEMPLETON, D.; CROCKER D. Determination of Structural Carbohydrates and Lignin in Biomass, Laboratory Analytical (LAP) - Technical Report, NREL/TP-510-42618, 2008a.

SLUITER, A.; RUIZ, R.; SCARLATA, C.; SLUITER, J.; TEMPLETON, D. Determination of Extractives in Biomass, Laboratory Analytical Procedure (LAP) - Technical Report, NREL/TP510-42619, 2008b.

VÁSQUEZ, M. P.; SILVA, J. N. C.; SOUZA JR., M. B.; PEREIRA JR., N. Appl. Biochem.and Biotechnol. 136-140, 141-154, 2007. 